\title{
Copépodes Harpacticoïdes cavernicoles de Bulgarie 2. Deux nouveaux représentants du genre Stygoelaphoidella Apostolov, 1985 du Nord-Ouest de la Bulgarie
}

\author{
A. Apostolov 1
}

Mots clés : Taxonomie, Copepoda, Harpacticoida, Stygoelaphoidella, Bulgarie.

Deux nouvelles espèces d'Harpacticö̈des, Stygoelaphoidella bulgarica n. sp. et $S$. elegans n. sp. découvertes dans les eaux cavernicoles de Bulgarie sont décrites.

Cave-dwelling harpacticoid copepods from Bulgaria 2. Two new Stygoelaphoidella Apostolov, 1985 from the Nord-West of Bulgaria

Keywords : Taxonomy, Copepod, Harpacticoida, Stygoelaphoidella, Bulgaria.

Two new species of Harpacticoida : Stygoelaphoidella bulgarica n.sp. and $S$. elegans $n$.sp. discovered from cave waters of Bulgaria are described.

Les recherches intensives sur le peuplement de différentes grottes de Bulgarie ont permis de récolter de nouveaux exemplaires du genre Stygoelaphoidella Apostolov, 1985 (Apostolov 1989, 1990). Depuis, à notre connaissance, aucune autre espèce de ce genre n'a été signalée.

Dans le cadre d'une étude faunistique des eaux cavernicoles du nord-ouet du la Bulgarie, M. Ivan Pandoursky, de l'Institut de Zoologie de Sofia, a recueilli une abondante collection de Copépodes Harpacticoïdes dont il a bien voulu me confier l'étude. Dans ce matériel, deux autres espèces du genre Stygoelaphoidella ont été découvertes.

Le présent travail est consacré à la description de ces espèces nouvelles; elles ont été récoltées dans la grotte «Drachanska » (nord-ouest de la Bulgarie) et près du village d'Iskretz en 1989.

\section{Stygoelaphoidella bulgarica n.sp. (fig. 1-4)}

Matériel examiné : 7 femelles et 2 mâles.

Localité : lac dans la grotte « Drachanska 》 près de la ville de Vratza (locus typicus) ; le 8 avril 1989 ( 8 $Q$ et $2 \sigma^{\circ} \sigma^{\circ}$ ) ; ruisseau dans cette grotte, le 8 avril 1989 (1 ९).

1. Izgrev, B1. 35, Bx. R. Burgas, 8008 Bulgarie.
Holotype : 1 femelle.

Paratype : I femelle et 1 mâle (collection de l'auteur).

\section{DESCRIPTION DE LA FEMELLE}

Segments du métasome sans ornementation. Urosome avec ornementation complexe. Segment génital sans ligne de suture médiane marquée ; champ génital (fig. 1 a) comme sur la figure. Avant-dernier segment avec une rangée ininterrompue de spinules au bord distal et ventral. Dernier segment avec, sur la face ventrale, quatre spinules à la base de chaque branche furcale (fig. 1 b).

Dernier segment abdominal sans rangée de spinules dorsales. Bord libre de l'opercule anal avec 13-14 petites spinules. Branches furcales légèrement plus longues que larges ; elles portent deux soies apicales bien développées, une courte soie apicale interne, une soie subapicale externe, deux soies latérales et une soie, articulée à sa base, sur la face dorsale (fig. $1 \mathrm{c}$ ).

Antennule (fig. 1 d) : composée de huit articles. Les trois premiers articles sont allongés ; le premier porte une soie apicale. La chétotaxie des autres articles est indiquée sur la figurc. Aesthétasque et soie accompagnatrice sur le quatrième article. L'avant- 
dernier article et le dernier portent, également, des soies articulées à leur base en position externe.

Antenne (fig. 1 e) : coxa courte et nue. Allobasis plus long que l'endopodite, portant une soie interne, barbelée. Exopodite uni-articulé avec quatre addendes. Endopodite armé de spinules, de trois forts crochets et de quatre soies géniculées.

$\mathrm{P}_{1}$ (fig. 2 a): basis avec deux épines, l'unc externe, forte, l'autre interne, plus mince. Premier article de l'endopodite aussi long que les deux premiers articles de l'exopodite ; il porte une épine au tiers distal de son bord interne; deuxième article avec une soie barbelée ; troisième article avec trois soies inégales. Exopodite avec une épine à l'angle distal externe des deux premiers articles et au bord interne du deuxième. Article terminal avec quatre soies.

$\mathrm{P}_{2}$ (fig. $2 \mathrm{~b}$ ) : basis avec une épine au bord externe. Premier article de l'endopodite court, avec une soie interne; deuxième article avec deux soies internes, deux soies apicales et une épine subapicale externe. Exopodite triarticulé. Deuxième et troisième articles de l'exopodite avec une soie interne. Dernier article de l'exopodite avec cinq addendes. Exopodite avec les épines usuelles à l'angle distal externe des deux premiers articles.

$\mathrm{P}_{3}$ (fig. $2 \mathrm{c}$ ) : basis avec une longue épine externe. Endopodite biarticulé ; premier article court, avec une soie à l'angle distal interne; deuxième article avec deux épines au bord interne, deux soies apicales et une épine subapicale externe. L'endopodite n'atteint pas l'extrémité distale du deuxième article de l'exopodite. Exopodite triarticulé, avec les épines usuelles au bord externe des deux premiers articles et au bord interne du deuxième. Article terminal avec six addendes.

$\mathrm{P}_{4}$ (fig. $2 \mathrm{~d}$ ) : basis avec une longue soie externe. Endopodite uniarticulé, avec trois soies. Exopodite triarticulé ; premier article sans soie à l'angle distal interne ; deuxième article avec une longue soie au bord interne et troisième article avec six addendes.

Chétotaxie des $\mathrm{P}_{2}-\mathrm{P}_{4}$ :

$$
\text { Exp. }
$$$$
\text { Enp. }
$$

\begin{tabular}{|c|c|c|c|c|}
\hline$P_{2}$ & 0 & 1 & 122 & 1 \\
\hline$P_{3}$ & 0 & 1 & 222 & 1 \\
\hline 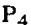 & 0 & 1 & 222 & - \\
\hline
\end{tabular}

$\mathrm{P}_{5}$ (fig. $2 \mathrm{e}, \mathrm{f}$ ) : baséoendopodite ne dépassant pas l'extrémité distale de l'exopodite ; il est armé de trois épines. Exopodite court avec une épine apicale bien développée, flanquée de deux épines plus courtes.

\section{DESCRIPTION DU MÂLE}

La morphologie générale est identique à celle de la femelle. Le dimorphisme sexuel porte sur l'antennule, les endopodites $\mathrm{P}_{2}-\mathbf{P}_{4}$, l'exopodite $\mathrm{P}_{4}$ et la $\mathbf{P}_{5}$.

Antennules subchirocères, composées de huit articles. Le premier article avec une soie. Le quatrième article porte l'aesthétasque principal et le distal six soies (fig. 3 a).

Antennes : comme chez la femelle.

$P_{1}$ (fig. 3 b) : comme chez la femelle.

$\mathrm{P}_{2}($ fig. $3 \mathrm{c}$ ) : la chétotaxie de l'exopodite est identique à celle de la femelle. Endopodite biarticulé ; premier article avec une courte soie interne ; deuxième article avec deux soies au bord interne et deux soies apicales barbelées. L'article distal de l'endopodite avec les épines usuelles à l'angle distal externe.

$\mathbf{P}_{3}$ (fig. $\left.3 \mathrm{~d}\right)$ : endopodite triarticulé : premier article glabre; deuxième article avec une longue épine lancéolée ; troisième article avec deux épines apicales. Troisième article de l'exopodite avec deux longues épines externes.

$\mathbf{P}_{4}$ (fig. 3 e) : endopodite uniarticulé avec une épine courte à son bord interne, une épine subapicale et une épine apicale. L'endopodite avec les épines usuelles à l'angle externe. Exopodite triarticulé, avec une soie interne au second article ; article distal avec six épines modifiées.

$\mathrm{P}_{5}$ (fig. $3 \mathrm{f}$ ) : baséoendopodite réduit à une lame chitineuse sans soie à sa partie interne. Exopodite petit avec trois épines, la médiane beaucoup plus développée que les autres.

Longueur de la femelle : $0.51 \mathrm{~mm}$.

Longueur du mâle : $0.50 \mathrm{~mm}$.

\section{DiAgNOSE}

\section{Femelle}

Corps allongé. Le rostre est très réduit. L'opercule anal avec 14 petites spinules. Branches furcales plus longues que larges. Antennule de huit articles. Antenne avec exopodite uniarticulé, portant 
(3)

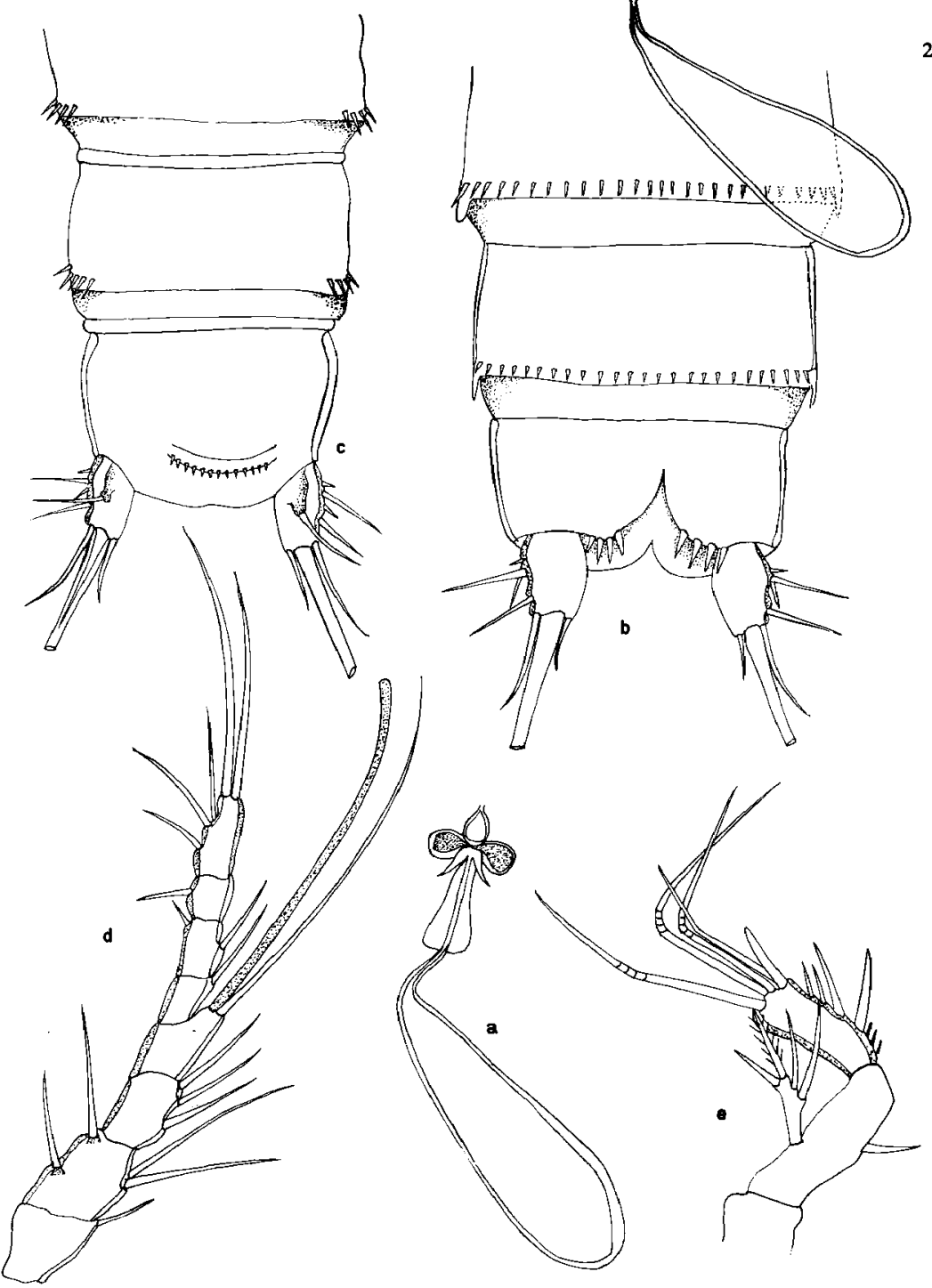




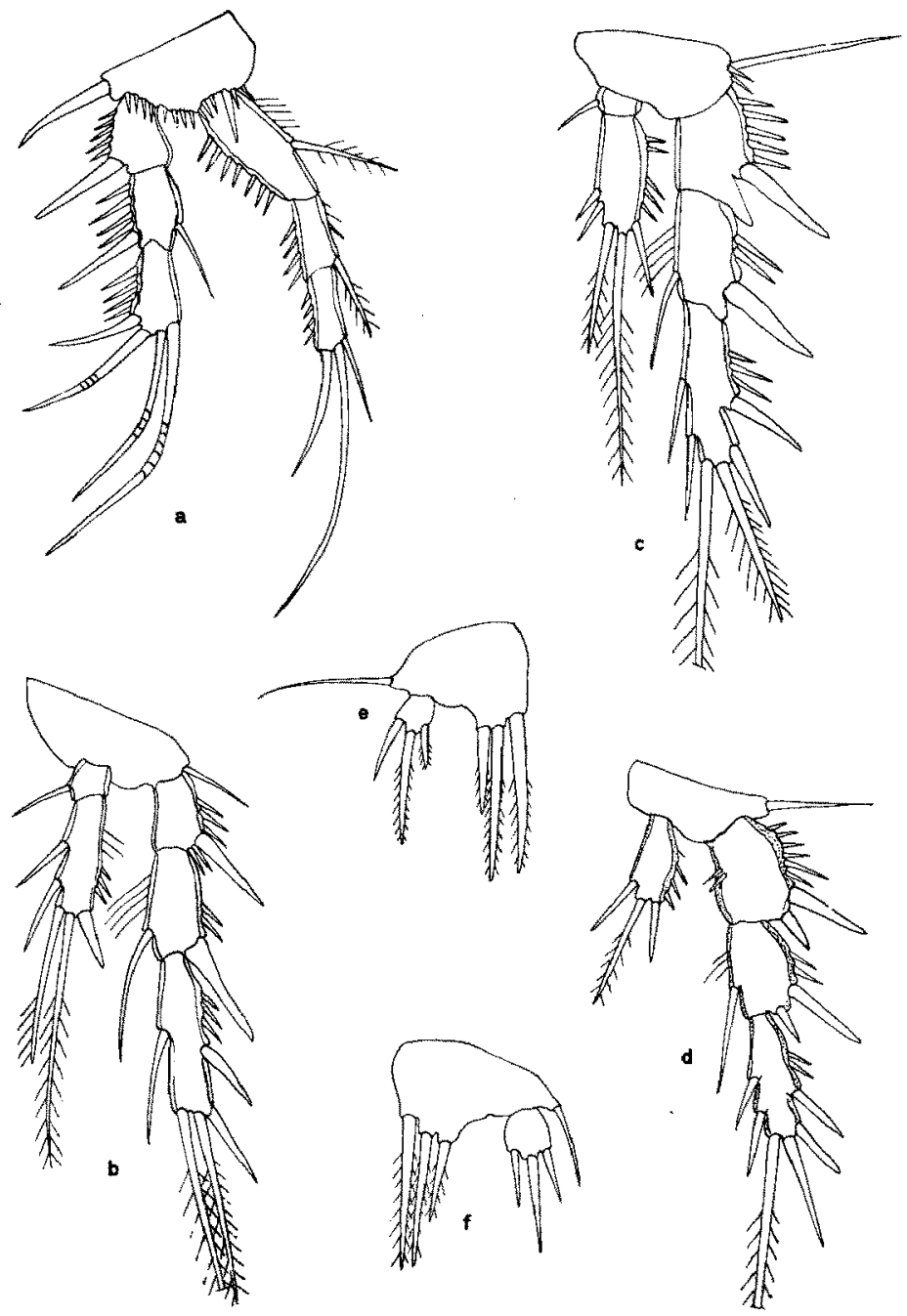

Fig. 2. Stygoelaphoidella bulgarica n. sp., $Q_{\text {. }} a: P_{1} ; b: P_{2} ; c: P_{3} ; d: P_{4} ; e: P_{5} ; f: P_{3}$. 
(5)

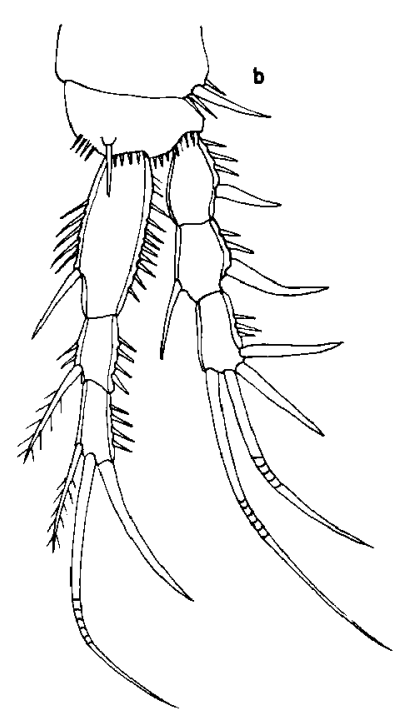

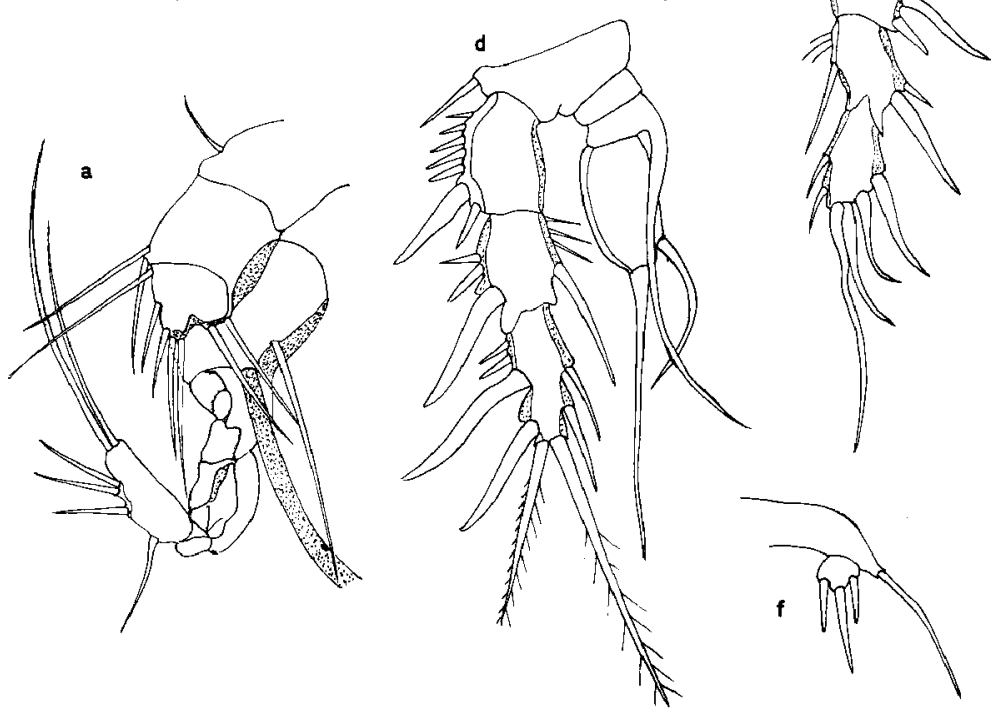
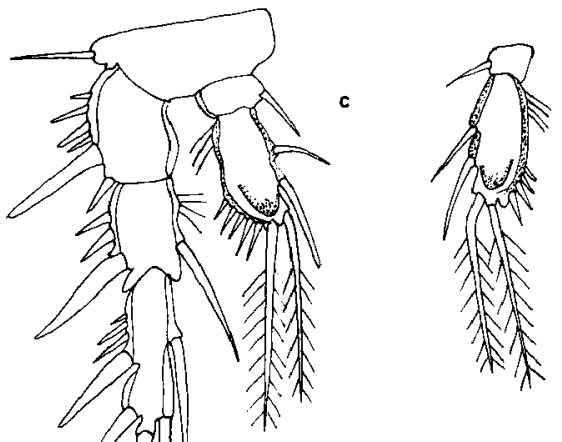

ANi

A

NA

$\mathbb{A}$

Fig. 3. Stygoelaphoidella bulgarica n.sp., $\sigma: a: \mathbf{A}_{1} ; \mathrm{b}: \mathbf{P}_{1}: \mathfrak{c}: \mathrm{P}_{2} ; \mathrm{d}: \mathrm{P}_{3}: \mathrm{e}: \mathrm{P}_{4} ; f: \mathrm{P}_{5}$. 

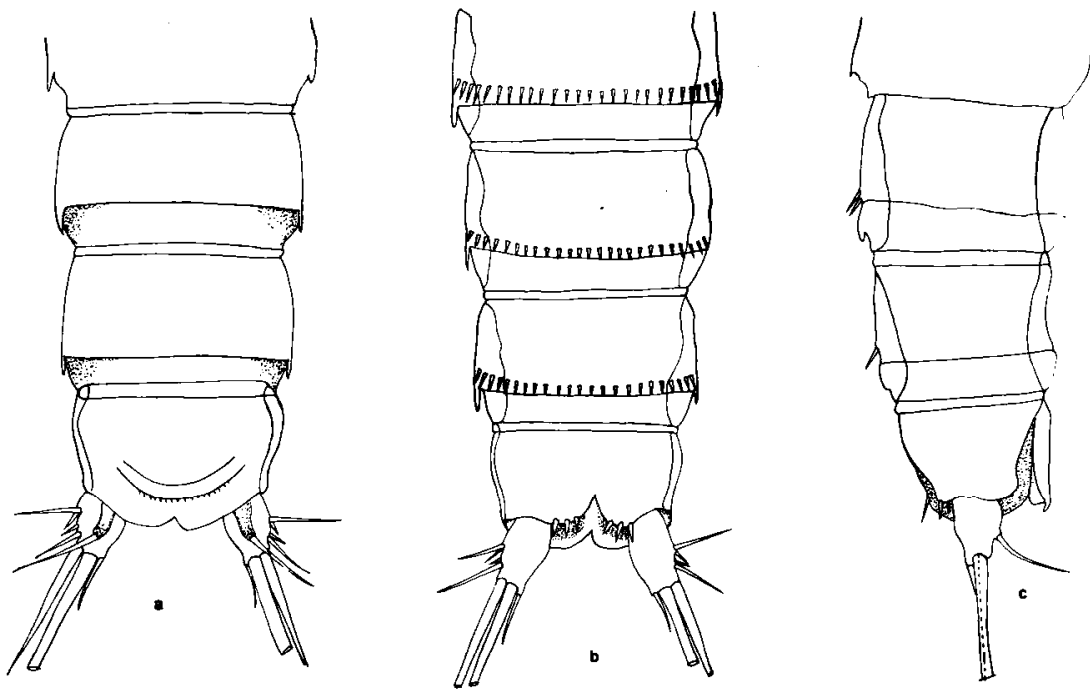

Fig. 4. Stygoelaphoidella bulgarica n.sp., $\sigma$ : a : furca, vue dorsale ; b : furca, vue ventrale ; c : furca, vue latérale.

quatre épines. Premier article de l'exopodite des $\mathrm{P}_{2}-\mathrm{P}_{4}$ sans soie interne, article médian avec une soie interne, article distal avec 5,6 et 6 soies. Baséoendopodite de la $\mathrm{P}_{5}$ avec trois soies ; exopodite ovale avec trois soies.

\section{Mâle}

Antennule subchirocère. Article distal de l'endopodite $\mathrm{P}_{2}$ transformé. Endopodite $\mathrm{P}_{3}$ triarticulé ; article médian avec une longue épine lancéolée. Endopodite $\mathrm{P}_{\mathbf{4}}$ uniarticulé avec trois épines modifiées ; troisième article de l'exopodite avec six épines modifiées. Baséoendopodite de la $P_{5}$ réduit à une lame chitineuse, sans soie à sa partie interne ; exopodite ovale avec trois épines.

\section{DisCussion}

Stygoelaphoidella bulgarica n.sp. se montre originale par la chétotaxie de ses pattes thoraciques et, notamment, par la présence d'une soie interne au premier article de l'endopodite $P_{3}$ de même que par la forme des branches furcales. La nouvelle espèce présente de très fortes affinités avec Stygoelaphoidella subterranea Apostolov, 1990 ; ce sont les deux seules espèces de Stygoelaphoidella qui portent une soie interne à l'article basal de l'endopodite de la $\mathrm{P}_{3}$. Stygoelaphoidella bulgarica n.sp. se distingue cependant aisément de $S$. subterranea par la morphologie très particulière de l'endopodite des $\mathrm{P}_{2}-\mathbf{P}_{4}$ des mâles et par l'aspect des soies qui arment l'endopodite $\mathrm{P}_{\mathbf{4}}$ et l'article distal de l'exopodite $\mathbf{P}_{\mathbf{4}}$.

Stygoelaphoidella elegans n.sp. (fig. 5-7)

Matériel examiné : 19 femelles et 8 mâles.

Localité : Mare dans la grotte « Marina doupka » près du village Brézé (locus typicus) ; le 28 janvier 
1989 (4 \& $\odot$ et 3 o o ). Grotte "Tzarkveto " près du village Iskretz; le 22 février 1989 (15 $Q Q$ et $\left.50^{\circ} 0^{\circ}\right)$.

Holotype : 2 femelles.

Paratypes : 1 femelle et 1 mâle (collection de l'auteur).

\section{DESCRIPTION DE LA FIMELLE}

Segment génital sans ligne de suture médiane marquée, même latéralement. Segments de l'urosome sans ornementation dorsale ; ventralement, le segment génital et les deux suivants portent une rangée de spinules (fig. 5 a). Segment anal avec trois épines ventrales à la base de chaque branche furcale. Branches furcales rectangulaires à leur base, s'amincissant à leur partie distale ; elles portent trois soies apicales dont l'interne peu développée, la médiane globuleuse à sa base et deux soies latérales au bord externe, chacune avec une épine à sa base (fig. $5 \mathrm{~b}$ ).

Antennule (fig. 5 e) : à huit articles dont le quatrième avec un aesthétasque.

Antenne (fig. $5 \mathrm{f}$ ) : allobasis glabre. Exopodite uniarticulé avec quatre addendes. Endopodite avec trois épines et trois spinules à sa face antérieure, quatre longues soies et une épine à l'apex.

$P_{1}$ (fig. 6 a): basis avec deux épines, l'une externe, l'autre interne. Premier article de l'endopodite atteignant l'extrémité distale du deuxième article de l'exopodite; il est armé d'une soie à l'angle distal interne; deuxième article avec une soie barbelée distale interne ; troisième article avec trois addendes. Exopodite triarticulé avec une forte épine à l'angle distal externe des deux premiers articles ; troisième article avec quatre addendes ; deuxième article avec une soie distale interne. Exopodite avec les épines usuelles à l'angle distal externe des trois articles.

$P_{2}$ (fig. $6 \mathrm{~b}$ ) : basis avec une forte épine externe. Exopodite triarticulé ; angle distal externe des deux premiers articles armé d'une forte épine et de plusieurs spinules; bord interne du deuxième article avec une courte épine et quatre spinules ; troisième article avec une épine interne, deux soies apicales, une épine subapicale et une épine externe. Endopodite biarticulé; premier article avec une soie interne ; deuxième article avec une ou deux soies au bord interne, deux soies apicales et une épine subapicale externe.
$\mathrm{P}_{3}$ (fig. $6 \mathrm{c}$ ) : basis avec une longue soie externe. Exopodite triarticulé ; angle distal externe des deux premiers article armé d'une forte épine et de nombreuses spinules ; bord interne du deuxième article avec une courte épine ; troisième article avec deux soies internes, une soie et une épine apicales et deux épines externes. Endopodite biarticulé ; premier article court avec une épine interne; deuxième article avec deux soies au bord interne, deux épines apicales et une épine subapicale externe.

$\mathrm{P}_{4}(\mathrm{fig} .6 \mathrm{~d}$ ) : basis avec une courte épine externe. Endopodite uniarticulé avec trois soies. Exopodite triarticulé ; article distal avec six addendes.

La chétotaxie des $\mathrm{P}_{2}-\mathrm{P}_{4}$ peut être résumée ainsi :

\begin{tabular}{|c|c|c|c|c|c|}
\hline & & & Exp. & & Enp. \\
\hline & 0 & 1 & 122 & 1 & 221 \\
\hline $\mathbf{P}_{3}$ & 0 & 1 & 222 & 1 & 22 \\
\hline & 0 & 1 & 222 & . & 111 \\
\hline
\end{tabular}

$P_{5}(f i g .6 \mathrm{e}):$ baséoendopodite atteignant la moitié de la longueur de l'exopodite ; il porte trois épines dont l'externe est la plus courte. Exopodite ovale, avec trois épines apicales.

\section{DESCRIPTION DU MĀLE}

La chétotaxie de $P_{1}$ et $\mathbf{P}_{4}$, des exopodites $P_{2}$ et $P_{3}$ comme chez la femelle.

$\mathrm{P}_{2}$ (fig. $7 \mathrm{~b}$ ) : endopodite biarticulé ; premier article avec une épine à l'angle distal interne ; deuxième article avec deux épines au bord interne et deux épines apicales.

$P_{3}$ (fig. $7 \mathrm{c}$ ) : basis avec une longue soie externe. Endopodite triarticulé ; premier article avec une soie au bord interne ; deuxième article avec une longue apophyse lancéolée ; troisième article avec deux soies apicales.

$\mathrm{P}_{4}$ (fig. $7 \mathrm{~d}$ ) : comme chez la femelle.

$\mathrm{P}_{5}$ (fig. $7 \mathrm{e}$ ) : baséoendopodite réduit à une lame chitineuse, sans soies à sa partie interne. Exopodite avec trois épines dont l'interne est la plus longue.

Longueur de la femelle : $0,51 \mathrm{~mm}$.

Longueur du mâle : $0,38-40 \mathrm{~mm}$.

\section{DISCUSSION}

La nouvelle espèce ressemble, d'après la clé de détermination des espèces du genre Stygoelaphoidella d'Apostolov (1989), à Stygoelaphoidella 

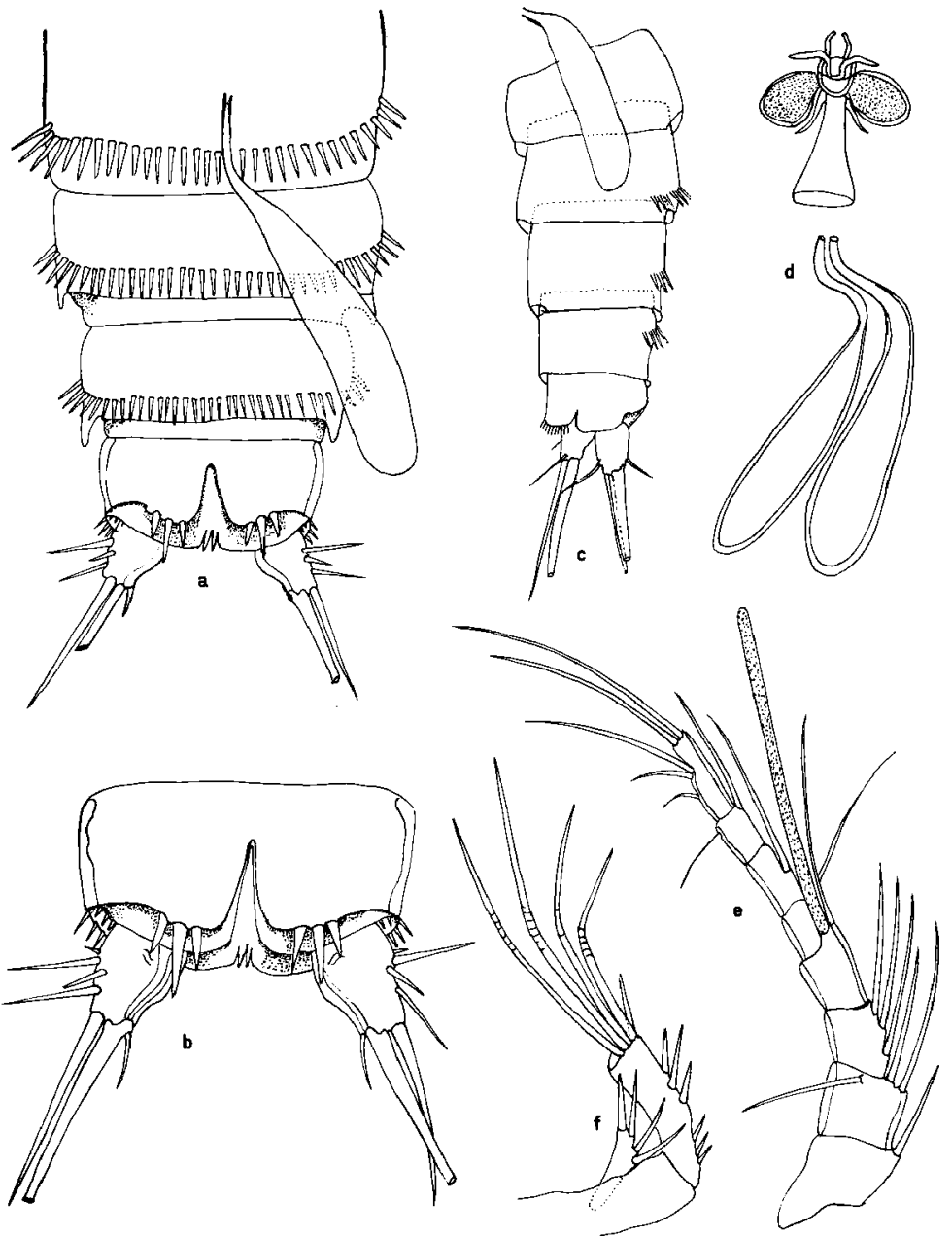

Fig. 5. Stygoelaphoidella elegans n. sp., ○. a : urosome, vue ventrale ; b : furca, vue ventrale ; $\mathrm{c}$ : urosome, vue latérale; $d$ : aire génitale ; e : $A_{1} ; f: A_{2}$. 


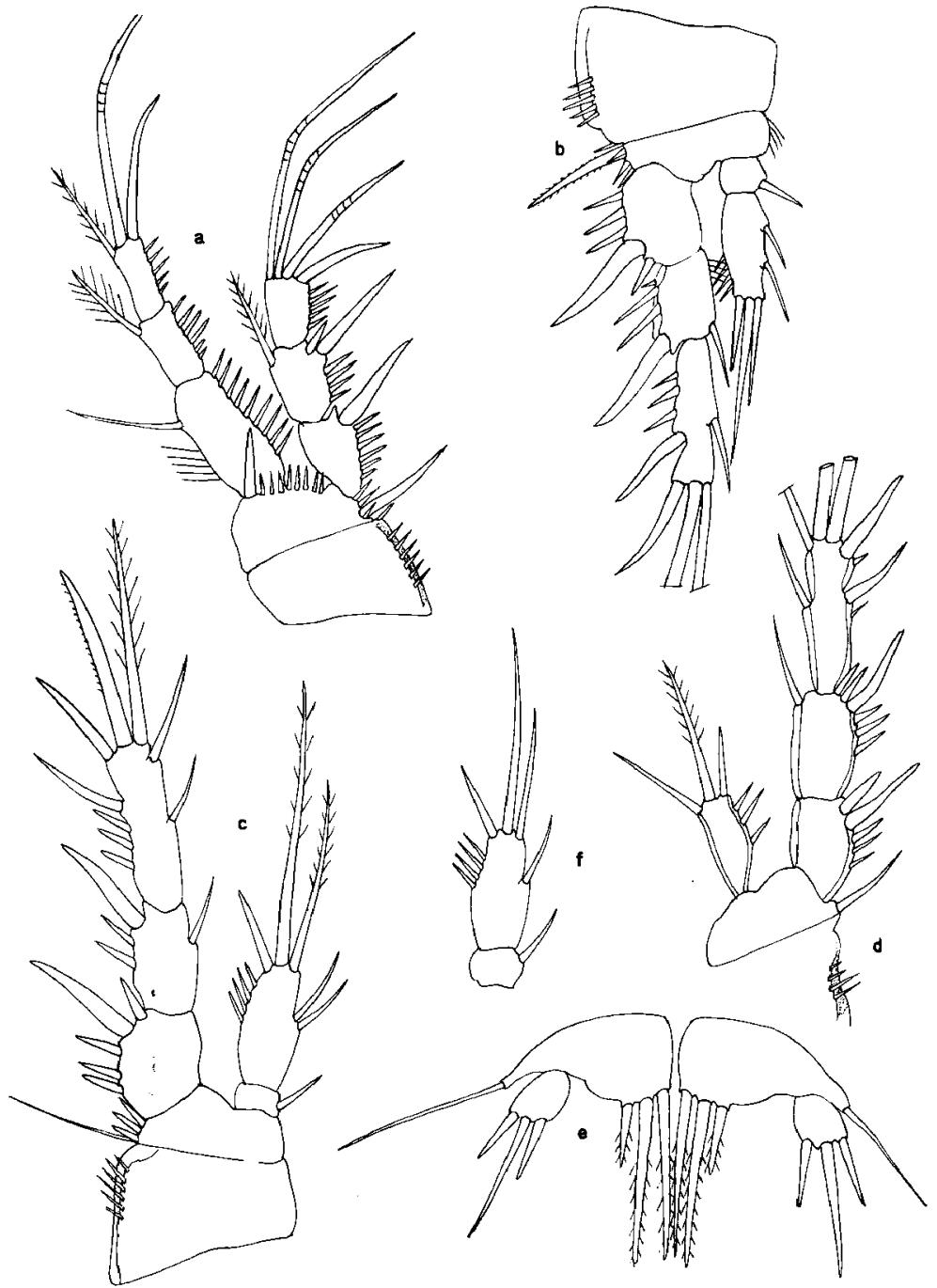

Fig. 6. Stygoelaphoidella elegans n.sp., $Q: a: P_{1} ; b: P_{2} ; c: P_{3} ; d: P_{4} ; e: P_{5} ; f:$ Enp. $P_{2}$. 


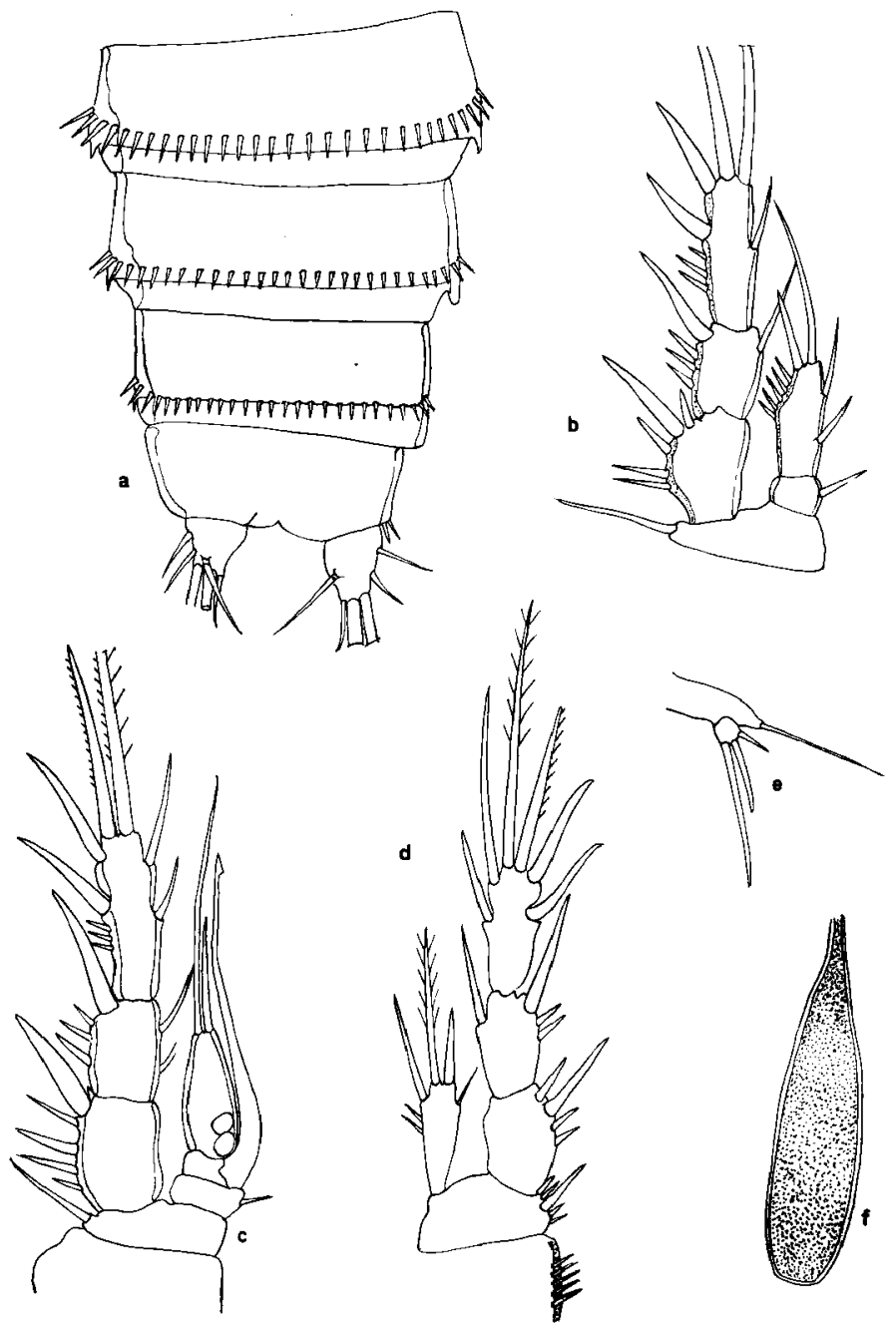

Fig. 7. Stygoelaphoidella elegans $n$. sp., o. a : furca, vue ventrale; $b: P_{2} ; c: P_{3} ; d: P_{4} ;$ e $: P_{5} ; f:$ spermatophore. 
subterranea et $S$. bulgarica n.sp., toutes deux connues de Bulgarie seulement. Elle s'en distingue cependant par plusieurs points : l'ornementation et la longueur des branches furcales ; la configuration des péréiopodes 5 ; l'ornementation de l'urosome de la femelle et du mâle ainsi que celle du dernier segment et de l'opercule anal.

\section{Remerciements}

Je remercie M. Ivan Pandoursky qui à effectué le tri des récoltes et qui a bien voulu me confier l'étude des Copépodes Harpacticoïdes des eaux cavernicoles bulgares.

\section{Travaux cités}

Apostolov (A.). 1989. - Description d'un nouveau représentant du genre Stygoelaphoidella Apostolov, 1985 (Copepoda, Harpacticoida) dans les eaux cavernicoles de Bulgarie. Annis Limnol., 25 (2) : $101-106$.

Apostolov (A.). 1990. - Les Harpacticoides (Copepoda) des eaux souterraines de Bulgarie. Boll. Mus. civ. St. nat. Verona, I(sous presse). 